\title{
Evolução da disponibilidade domiciliar de alimentos no município de São Paulo no período de 1979 a 1999
}

\author{
Evolution of household food availability in the \\ municipality of São Paulo from 1979 to 1999
}

Rafael Moreira CLARO'

Flávia Mori Sarti MACHADO2

Daniel Henrique BANDONI ${ }^{3}$

RE S U M O

\section{Objetivo}

Analisar a evolução (1971-1999) da disponibilidade domiciliar de alimentos e nutrientes no município de São Paulo.

\section{Métodos}

O estudo utiliza dados de duas Pesquisas de Orçamentos Familiares, conduzidas pela Fundação Instituto de Pesquisas Econômicas, durante os períodos de 1971-1972 (2.380 domicílios) e 1998-1999 (2.351 domicílios) na região urbana do município de São Paulo. A disponibilidade diária per capita de alimentos foi estimada pelo cálculo da fração da quantidade total de alimentos adquirida em um mês, atribuível a cada indivíduo participante na pesquisa, com posterior divisão pelo número de dias do mês. O padrão alimentar da população foi caracte-rizado de acordo com a participação relativa de 18 grupos de alimentos e nutrientes na aquisição do total de alimentos nos respectivos anos.

\section{Resultados}

Os resultados mostram aumento no consumo de carnes, lácteos, alimentos processados e refeições prontas. Por outro lado, observou-se redução no consumo de cereais, leguminosas, frutas e hortaliças. O consumo de açúcar, óleos e gorduras apresentou leve redução, entretanto, considerando-se a elevação no consumo de alimentos processados observada, não há como caracterizá-la como melhoria na qualidade da dieta. Em paralelo, observou-se aumento no consumo de proteínas, gorduras e cálcio; enquanto o consumo de carboidratos, fibras, folato e vitamina $C$ apresentou redução.

\footnotetext{
1 Universidade de São Paulo, Núcleo de Pesquisas Epidemiológicas em Nutrição e Saúde. Av. Dr. Arnaldo, 715, Cerqueira César, 01246-904, São Paulo, SP, Brasil. Correspondência para/Correspondence to: R.M. CLARO. E-mail: <rclaro@usp.br>.

2 Universidade de São Paulo, Escola de Artes, Ciências e Humanidades. São Paulo, SP, Brasil.

3 Universidade de São Paulo, Faculdade de Saúde Pública, Departamento de Nutrição. São Paulo, SP, Brasil.
} 
484 | R.M. CLARO et al.

\section{Conclusão}

As mudanças observadas no padrão alimentar da população de São Paulo são consistentes com os crescentes índices de prevalência de obesidade e doenças crônicas não-transmissíveis observados no município.

Termos de indexação: consumo de alimentos; dieta; inquéritos nutricionais.

\section{A B S T R A C T}

\section{Objective}

Food budget surveys data were analyzed in order to describe the evolution (1971-1999) of food and nutrient availability in São Paulo, Brazil.

\section{Methods}

The study uses data from two Family Budget Surveys conducted by Fundação Instituto de Pesquisas Econômicas, carried out during the periods of 1971-1972 (2,380 households) and 1998-1999 (2.351 households) in the urban area of São Paulo city. Per capita daily food availability was estimated by calculating the fraction of all the food bought for the month attributed to each participant of this study divided by the number of days in the month. The food patterns of this population were characterized according to the caloric and nutrient distribution of the 18 food groups and nutrients obtained in the respective surveys.

\section{Results}

The results show increased consumption of meats, dairy, processed foods and chilled and frozen meals. On the other hand, the consumption of grains, fruits and vegetables decreased. The consumption of sugar, oils and fats reduced slightly but since the consumption of processed foods increased, it is not possible to say that diet quality improved. At the same time the consumption of proteins, fats and calcium increased while the consumption of carbohydrates, fiber, folate and vitamin $C$ decreased.

\section{Conclusion}

The changes in the eating patterns of the population of São Paulo are consistent with the growing prevalence of obesity and non-communicable chronic diseases observed in the city.

Indexing terms: food consumption; diet; nutritional surveys.

\section{N T R O D U Ç Ã O}

Ao longo das últimas décadas, os índices de prevalência de obesidade e doenças crônicas não transmissíveis (DCNT) associadas à dieta têm se elevado em ritmo acelerado, tanto em países desenvolvidos quanto nos em países em desenvolvimento' ${ }^{1}$. Embora diversos fatores influenciem o panorama epidemiológico, mudanças recentes na alimentação, associadas ao processo de transição nutricional, desempenham papel central nesse cenário'. O ambiente urbano atual conduz a uma ingestão cada vez maior de energia, enquanto limita a prática de atividades físicas ${ }^{2,3}$. Esse quadro é especialmente importante em vista das estimativas atuais dos altos custos que a obesidade e as DCNT impõem tanto aos sistemas de saúde nacionais quanto ao bem-estar e qualidade de vida dos indivíduos ${ }^{4}$.
Estudos sobre a tendência de mudanças no padrão alimentar da população brasileira destacam a elevação do consumo de carnes e alimentos industrializados (refrigerantes, biscoitos e refeições prontas) e a redução do consumo de leguminosas, raízes e tubérculos, frutas, legumes e verduras $(F L V)^{5}$. Evidências sugerem que a alta prevalência de doenças crônicas e obesidade poderia ser revertida pela inversão de tais tendências ${ }^{3}$. Além disso, o conhecimento atual indica que a adequação do consumo energético associada à prática freqüente de atividades físicas é, possivelmente, a única forma efetiva de combate à obesidade $^{3,6}$.

A utilização de inquéritos dietéticos para a investigação direta do consumo alimentar é a forma mais apropriada para caracterização dos padrões de alimentação de uma população? 
Entretanto, o custo para execução de formas confiáveis desse tipo de estudo (como, por exemplo, pesagem direta dos alimentos consumidos durante dado período) é elevado, enquanto a utilização de métodos menos dispendiosos para obtenção das informações nem sempre resulta em estimativas confiáveis.

A ausência de inquéritos dietéticos confiáveis e abrangentes implica no uso de dados alternativos, como Pesquisas de Orçamento Familiar (POFs), para caracterização do padrão dietético de populações. As POFs são inquéritos domiciliares com informações detalhadas sobre gastos efetuados pelas unidades de consumo para aquisição de bens e serviços ${ }^{9}$. Embora não permitam a avaliação do consumo alimentar individual, podem ser utilizadas como instrumento de verificação da ocorrência de mudanças nos hábitos alimentares da população por meio da avaliação das variações nas quantidades per capita de alimentos adquiridos, sendo uma importante fonte de dados ${ }^{7}$.

A POF realizada pela Fundação Instituto de Pesquisas Econômicas da Universidade de São Paulo (FIPE-USP) desde a década de 1970 é uma importante base de dados para identificação de modificações no padrão de aquisição de alimentos da população do município de São Paulo. A realização periódica da POF-FIPE torna possível, ainda, analisar a evolução da disponibilidade domiciliar de alimentos e nutrientes no município de São Paulo entre 1971 e 1999, período marcante na evolução do padrão alimentar do município, sendo tal análise o principal objetivo do presente artigo.

\section{M É T O D O S}

As Pesquisas de Orçamentos Familiares realizadas pela Fundação Instituto de Pesquisas Econômicas, nos períodos de agosto de 1971 a setembro de 1972 e maio de 1998 a junho de 1999, são as bases de dados utilizadas no presente estudo. Ambas as pesquisas apresentam universo de estudo idêntico, a região urbana do município de São Paulo (atualmente com cerca de 10 miIhões de habitantes). As amostras pesquisadas nas POFs-FIPE de 1971-1972 e 1998-1999 foram de, respectivamente, 2.380 domicílios (10.418 indivíduos) e 2.351 domicílios (7.980 indivíduos) $)^{10,11}$.

A definição do tamanho da amostra da POF-FIPE 1971-1972, baseou-se em plano de amostragem de dois estágios por meio da extração de sub-amostras de uma amostra geral. Os estratos da amostra geral foram estabelecidos com base na estratificação geográfica do cadastro de endereços da empresa de distribuição de energia elétrica local, de forma que os domicílios fossem selecionados pelo método de probabilidade proporcional de seleção ${ }^{10}$. Já o plano de amostragem da POF-FIPE 1998-1999 baseou-se na distribuição de renda das famílias paulistanas (estimadas nas POFs 1981-1982 e 1990-1991), com sorteio dos domićlios realizado de forma direta, baseado em amplo cadastro constituído pelo cadastro da companhia de distribuição de energia local complementado por cadastro de famílias residentes em áreas faveladas (fornecido pela Coordenadoria do Bem-Estar Social da Prefeitura Municipal) ${ }^{11}$.

A qualidade de alimentos disponível para consumo no domicílio, na POF 1971-1972, foi estimada com base na divisão do gasto mensal declarado pelas famílias com cada tipo de alimento pelo preço médio de varejo do alimento no mesmo estudo $^{10}$. Já a quantidade de alimento disponível, na POF 1998-1999, foi estimada diretamente a partir das quantidades de alimentos adquiridas declaradas pelas famílias. Em ambos os estudos, o total per capita diário de alimentos foi estimado pelo cálculo da fração da quantidade total de alimentos adquirida em um mês, atribuível a cada indivíduo participante na pesquisa, com posterior divisão pelo número de dias do mês (30 dias) ${ }^{11}$.

Os grupos de alimentos foram definidos com base na distribuição em grupos adotada no estudo nacional de orçamentos familiares mais recentemente concluído, a POF-IBGE 2002-2003, que distribui os alimentos nos seguintes grupos: Cereais e derivados; Feijões e outras leguminosas; 
Tubérculos e derivados; Carnes; Leites e derivados; Ovos; Frutas e sucos naturais; legumes, verduras; Óleos e gorduras vegetais; Gordura animal; Açúcar; Oleaginosas; Condimentos; Bebidas não-alcoólicas; e Refeições prontas e misturas industrializadas ${ }^{12}$. No contexto do presente estudo, os Alimentos Processados, originalmente distribuídos entre os demais grupos, passaram a integrar um grupo próprio, dividido em seis categorias: Doces; Refrigerantes (inclusive sucos industrializados artificiais); Embutidos; Cereais processados; Bolachas e biscoitos; e Molhos e caldos (inclusive maionese).

Foram atribuídos fatores de correção às aquisições de alimentos com base no banco de dados do Estudo Nacional da Despesa Familiar (ENDEF), a fim de excluir a parcela referente à parte não comestível dos alimentos ${ }^{13}$. A conversão da quantidade total de alimentos adquiridos em calorias e nutrientes foi realizada a partir de um aplicativo desenvolvido no Departamento de Nutrição da Faculdade de Saúde Pública da Universidade de São Paulo, que faz uso dos dados da tabela de composição de alimentos do ENDEF ${ }^{13}$.

Os registros de produtos sem especificação clara de nome nos diversos grupos de despesas alimentares (por exemplo, registro de fruta sem especificação) receberam valor energético baseado no valor médio dos demais alimentos do mesmo grupo. Os registros sem possibilidade de classificação em nenhum grupo de alimentos (por exemplo, Alimentos sem especificação) foram excluídos dos cálculos nutricionais.

A caracterização dos padrões alimentares de 1971-1972 e 1998-1999 no município, foi estabelecida com base na participação relativa de grupos de alimentos e alimentos de aquisição mais freqüente no total energético disponível. A descrição do aporte relativo de nutrientes na disponibilidade alimentar considerou a contribuição de macronutrientes, fibras, folato, vitamina C, sódio, cálcio e ferro. A participação relativa dos nutrientes na disponibilidade alimentar familiar foi expressa pelo teor do nutriente em mil calorias.

Foi feita análise descritiva dos dados, baseando-se nas análises realizadas anterior- mente, para identificar a tendência secular do padrão de disponibilidade domiciliar de alimentos no Brasil, obtida a partir das Pesquisas de Orçamentos Familiares do Instituto Brasileiro de Geografia e Estatística (IBGE), entre os anos de 1974-1975 e 2002-20035,7. Assim, a análise da disponibilidade no município de São Paulo foi realizada com base na média da aquisição per capita de grupos de alimentos no conjunto consolidado da amostra de domicílios pesquisados. A análise realizada a partir das POFs da FIPE permite expressar a evolução do padrão de consumo alimentar para o município de São Paulo, além disso, o dado pode ser comparado aos resultados obtidos nas POFs do IBGE, permitindo identificar a influência do acelerado processo de urbanização registrado no município, em contraposição à situação do padrão alimentar de outras regiões metropolitanas avaliadas em estudos prévios ${ }^{5,7}$.

\section{RESULTADOS}

A Tabela 1 apresenta a evolução da participação relativa de 16 grupos de alimentos nas pesquisas de 1971-1972 e 1998-1999. Observa-se tendência de queda em quase todos os grupos, enquanto o grupo dos alimentos processados e o grupo dos alimentos prontos e misturas industrializadas apresentou aumento expressivo no período de quase três décadas compreendido entre as duas pesquisas. As refeições prontas, que representavam baixo percentual de aquisição na década de 1970, também passaram a apresentar uma fatia significativa das calorias disponíveis, superior ao grupo das leguminosas. Verificou-se aumento na disponibilidade do grupo das carnes e grupo do leite e derivados.

A Figura 1 exibe a evolução de alimentos selecionados. Verifica-se queda da participação relativa no total calórico para a maioria dos alimentos, com destaque para as frutas e os alimentos básicos. Observou-se aumento no período para aves, suco de frutas naturais e margarina. 
Tabela 1. Participação relativa (\%) de grupos de alimentos na disponibilidade total de energia, entre 1971-1972 e 1998-1999. São Paulo. 2006

\begin{tabular}{lcccc}
\hline \multirow{2}{*}{ Grupo de alimentos } & \multicolumn{2}{c}{ \% Energia } & Variação absoluta (\%) & Variação relativa (\%) \\
\cline { 2 - 4 } & 1971-1972 & $1998-1999$ & & \\
\hline Cereais e massas & 34,4 & 27,1 & $-21,1$ & $-7,3$ \\
Leguminosas & 5,4 & 3,3 & $-38,2$ & $-2,1$ \\
Tubérculos & 1,2 & 0,8 & $-36,4$ & $-0,4$ \\
Carnes & 7,6 & 8,9 & 17,2 & 1,3 \\
Leites e derivados & 7,7 & 8,4 & 9,6 & 0,7 \\
Ovos & 1,8 & 0,9 & $-50,0$ & $-0,9$ \\
Frutas e sucos naturais & 5,7 & 3,6 & $-36,4$ & $-2,1$ \\
Legumes & 1,5 & 1,0 & $-33,2$ & $-0,5$ \\
Verduras & 0,5 & 0,2 & $-52,7$ & $-0,3$ \\
Óleos e gorduras vegetais & 15,0 & 14,7 & $-1,7$ & $-0,3$ \\
Óleos e gorduras animais & 2,3 & 0,7 & $-72,1$ & $-1,7$ \\
Açúcares & 12,0 & 10,1 & $-15,9$ & $-1,9$ \\
Nozes/oleaginosas & 0,5 & 0,3 & $-46,7$ & $-0,2$ \\
Condimentos & 0,1 & 0,2 & 98,8 & 0,1 \\
Alimentos prontos e misturas industrializadas & 0,1 & 3,8 & $5.158,0$ & 3,7 \\
Alimentos processados & 4,4 & 16,0 & 266,6 & 11,7 \\
\hline
\end{tabular}

Carnes, ovos e leites

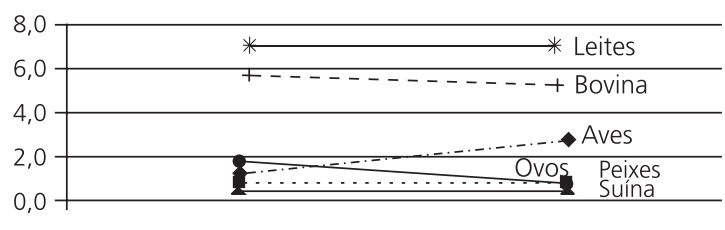

1971-1972 1998-1999

Alimentos básicos

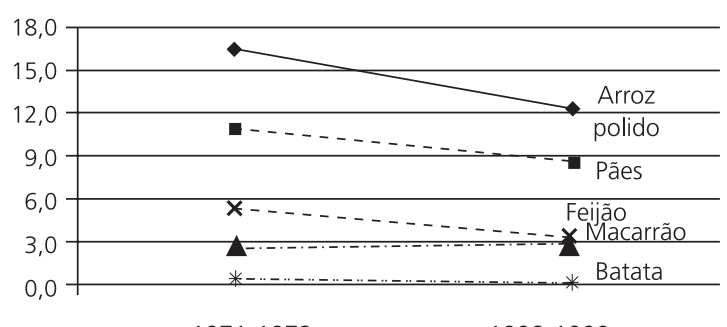

1971-1972

1998-1999

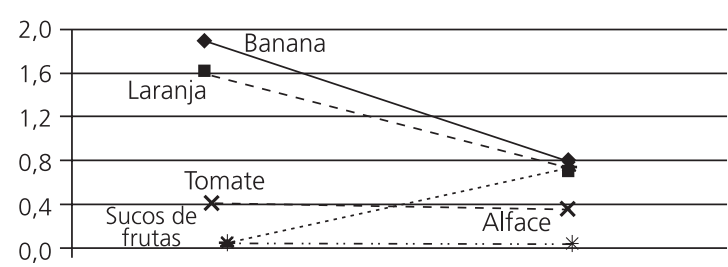

1971-1972

1998-1999

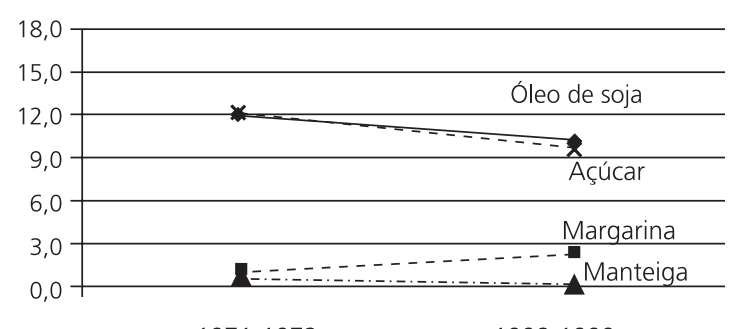

1971-1972

1998-1999

Figura 1. Participação relativa (\%) de alimentos selecionados na disponibilidade de energia, entre 1971-1972 e 1998-1999. São Paulo. 2006.

A evolução individual dos principais alimentos do grupo dos processados mostra uma tendência uniforme de aumento na participação energética dos componentes do grupo, com desta- que aos doces e refrigerantes (Figura 2). O conjunto dos biscoitos e bolachas, que apresentava participação inferior a $1 \%$ durante a década de 1970, também apresenta aumento expressivo no período em estudo. 
Em relação ao perfil nutricional da dieta (Tabela 2), observa-se elevação nas proteínas e gorduras, assim como significativo decréscimo na participação de fibras, folato e vitamina C, acompanhando a tendência decrescente da disponibilidade de frutas, legumes e verduras. Demonstra-se a inadequação da disponibilidade domiciliar dos macronutrientes em relação às recomendações nutricionais (10\%a 15\% das calorias de proteínas, $20 \%$ a $30 \%$ de gorduras e $55 \%$ a $75 \%$ de carboidratos). Ainda que a disponibilidade dos alimentos processados tenha aumentado de forma expressiva no período, a participação do sódio apresentou redução, enquanto a participação do ferro manteve-se estável no período.

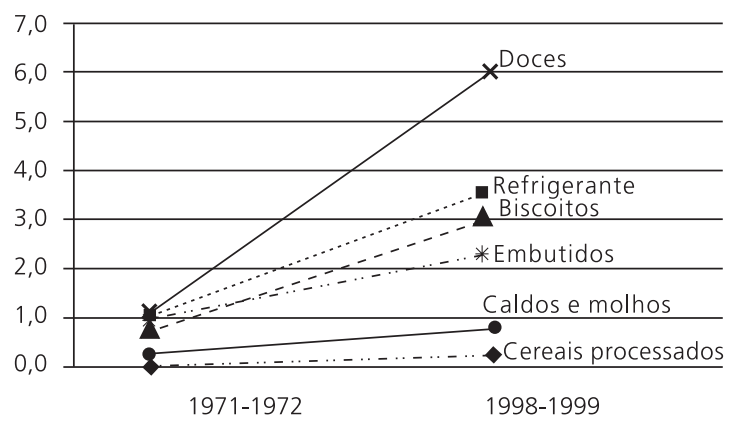

Figura 2. Participação relativa (\%) dos alimentos processados na disponibilidade total de energia entre 1971-1972 e 1998-1999, São Paulo. 2006.

Tabela 2. Tendência da participação de nutrientes na disponibilidade domiciliar de energia determinada pela aquisição alimentar, entre 1971-1972 e 1998-1999. São Paulo. 2006.

\begin{tabular}{|c|c|c|c|c|}
\hline \multirow{2}{*}{ Nutriente } & \multirow{2}{*}{ Unidade } & \multicolumn{2}{|c|}{ Nutriente por $1.000 \mathrm{kcal}$} & \multirow{2}{*}{$\begin{array}{l}\text { \% variação } \\
\text { do nutriente }\end{array}$} \\
\hline & & 1971-1972 & 1998-1999 & \\
\hline Proteínas & $g$ & 27,37 & 29,42 & 7,5 \\
\hline Carboidratos & $g$ & 141,65 & 133,71 & $-5,6$ \\
\hline Gorduras & $\mathrm{g}$ & 35,99 & 38,61 & 7,2 \\
\hline Proteínas & $\%$ & 10,95 & 11,77 & 7,5 \\
\hline Carboidratos & $\%$ & 56,66 & 53,48 & $-5,6$ \\
\hline Gorduras & $\%$ & 32,39 & 34,74 & 7,3 \\
\hline Fibras & G & 8,96 & 6,05 & $-32,4$ \\
\hline Folato & mcg & 97,33 & 67,43 & $-30,7$ \\
\hline Vitamina C & mg & 55,55 & 51,04 & $-8,1$ \\
\hline Sódio & $\mathrm{mg}$ & 2962,30 & 2243,35 & $-24,3$ \\
\hline Cálcio & $\mathrm{mg}$ & 316,89 & 398,66 & 25,8 \\
\hline Ferro & $\mathrm{mg}$ & 6,23 & 6,40 & 2,6 \\
\hline
\end{tabular}

\section{I S C U S S Ã O}

Os resultados obtidos demonstram a ocorrência de importantes mudanças no padrão de consumo alimentar paulistano no período de quase três décadas estudado. Verificou-se significativo declínio no consumo de alimentos básicos (cereais e derivados) e de frutas e hortaliças, enquanto o consumo de produtos de origem animal (carnes e laticínios) e alimentos processados apresentou grande expansão. De forma prática, tais resultados representam uma tendência desfavorável ao padrão de consumo alimentar em questão, com avanço da participação dos alimentos predominantemente industrializados, fontes de gorduras e açúcares. Conforme o esperado foi possível observar que o padrão de alimentação no município de São Paulo assemelha-se, cada vez mais, ao padrão ocidental de alimentação, freqüentemente relacionado como um dos agentes centrais da atual epidemia mundial de obesidade ${ }^{3}$.

A adequada análise dos dados apresentados requer a compreensão de que as informações contidas no presente estudo tratam da disponibilidade domiciliar de alimentos, não representando consumo alimentar propriamente dito, uma vez que se desconhece a fração dos alimentos adquirida que não tenha sido efetivamente consumida. Adicionalmente, as refeições realizadas fora do domicilio não foram suficientemente especificadas em nenhuma das POFs, sendo excluídas do presente estudo. No entanto, dados sobre a aquisição de alimentos mostram-se altamente correlacionados ao consumo real dos indivíduos ${ }^{14}$, além de fornecer informações úteis sobre o padrão alimentar das famílias em determinado período, permitindo estabelecer a participação relativa dos diferentes grupos e alimentos, que pode ser utilizada na determinação de políticas públicas que busquem modificar a oferta e o preço dos alimentos disponíveis à população?

O esforço em determinar um grupo dos alimentos processados se justifica na preocupação 
com a significativa expansão no consumo de tais alimentos, referida anteriormente em diversos estudos ${ }^{15,16}$. Assim, a criação de um grupo de alimentos que reúna apenas esta categoria tornou possível um melhor diagnóstico da evolução de seu consumo na cidade de São Paulo, cuja aquisição domiciliar apresentou-se inferior apenas à do grupo dos cereais na participação energética.

Embora o grupo dos açúcares apresente queda no período em questão, o considerável aumento no consumo de doces, refrigerantes e biscoitos e bolachas acarreta em aumento do consumo do açúcar adicionado aos alimentos e, possivelmente, não representa uma melhoria real de qualidade no padrão de consumo alimentar paulistano.

A análise da disponibilidade de grupos de alimentos apresentou alguns resultados esperados, como expressiva redução no consumo de gorduras animais e aumento no consumo de carnes e produtos lácteos. Outros resultados obtidos são bastante preocupantes, a redução no consumo dos grupos de frutas, verduras e legumes e cereais e leguminosas, gradativamente substituídos por alimentos adquiridos prontos (como refeições prontas) e alimentos processados, sendo os últimos detentores da maior carga de expansão na participação energética relativa durante o período estudado.

O amplo período compreendido entre as pesquisas, praticamente três décadas, possibilitou confirmar tendências de alimentação populacional importantes, como a substituição do consumo de manteiga por margarina, assim como a grande expansão na disponibilidade de alimentos processados, que registraram aumento de $500 \%$ para os doces, $300 \%$ para os refrigerantes e $400 \%$ para os biscoitos e bolachas, itens praticamente inexistentes nas prateleiras dos supermercados na década de 1970. A identificação de tal tendência demonstra a importância do estudo de pesquisas de orçamento familiar como instrumento de atuação em saúde pública, uma vez que o consumo de tais alimentos apresenta-se intimamente associado à ocorrência de obesidade e doenças crônicas associadas à dieta pela promoção de elevação no consumo de calorias oriundas de açúcares adicionados e gorduras trans.

Verificou-se, ainda, aumento na disponibilidade de alimentos de origem animal, como, por exemplo, carnes e leite. Apesar de a tendência apre-sentar características potencialmente positivas, evidenciadas pelo aumento no consumo de pro-teínas de alto valor biológico e cálcio, tais ali-mentos também constituem fontes de gordura animal e colesterol, nutrientes reconhecidamente danosos à saúde quando consumidos em quantidade excessiva.

Tendências de consumo alimentar semeIhantes à verificada ao longo do presente estudo também puderam ser constatadas em diversas áreas metropolitanas do Brasil em período de tempo semelhante, com destaque ao aumento da participação de carnes, embutidos, leite e derivados, refeições prontas e refrigerantes, associadas ao declínio de cereais, leguminosas e baixa participação de frutas e hortaliças 5,7,13.

A participação relativa dos nutrientes nas calorias demonstra a inadequação da dieta às recomendações nutricionais mais recentes, verificando-se aquisição de quantidades de gorduras superiores ao recomendado, em contraposição à participação de carboidratos inferior às recomendações, além de quantidade reduzida de fibras, demonstrando um desequilíbrio que pode contribuir para a ocorrência de DCNT e ganho de peso.

A tendência observada para participação dos nutrientes sugere um agravamento da inadequação registrada com a participação dos grupos e alimentos. $\mathrm{O}$ resultado é semelhante aos dados encontrados em diferentes áreas metropolitanas no Brasil, com aumento na participação das gorduras e proteínas e diminuição dos carboidratos ${ }^{13}$. Ainda assim, em São Paulo, há uma maior participação das gorduras totais e menor percentual energético de carboidratos. A participação do ferro se manteve praticamente estável, possivelmente devido ao aumento do consumo de alimentos de 
origem animal, fontes de ferro, como a carne bovina. Outro resultado interessante é a evolução negativa da quantidade de fibras e vitaminas (principalmente vitamina $\mathrm{C}$ e folato), possivelmente relacionada à diminuição na participação de frutas, verduras e legumes. Demonstra-se, portanto, que a evolução na disponibilidade domiciliar de alimentos tem conduzido a um padrão inadequado de alimentação.

Enfim, a tendência observada do padrão alimentar para a população paulistana não difere dos resultados registrados para a população brasileira de forma geral, sendo, ainda, compatível com a evolução na prevalência de sobrepeso e obesidade e de doenças crônicas não transmissíveis. Espera-se que a análise da evolução dos padrões alimentares da população paulistana propicie subsídios suficientes à melhor compreensão dos processos de transição epidemiológica e nutricional.

\section{COLABORADORES}

R.M. CLARO; F.M.S. MACHADO e D.H. BANDONI participaram no levantamento, organização, análise e interpretação dos dados, na redação e revisão do artigo.

\section{REFERÊNCIAS}

1. Popkin BM. The Nutrition transition: an overview of world patterns of change. Nut Rev. 2004; 62:s140-s3.

2. Hill JO, Sallis JF, Peters JC. Economic analysis of eating and physical activity: a next step for research and policy change. Am J Prev Med. 2004; 23: 111-6.

3. World Health Organization. Diet, nutrition and the prevention of chronic diseases. Geneva; 2003. WHO-Technical Report Series, 916.
4. Seidell JC. The impact of obesity on health status: some implications for health care cost. Int J Obes. 1995; 19(Suppl 6):s13-s6.

5. Levy-Costa RB, Sichieri R, Pontes NS, Monteiro CA. Disponibilidade domiciliar de alimentos no Brasil: distribuição e evolução (1974-2003). Rev Saúde Pública. 2005; 39(4):530-40.

6. Lock K, Pomerleau J, Causer L, Altmann DR, Mc-Kee M. The global burden of disease attributable to low consumption of fruit and vegetables: implications for the global strategy on diet. Bull World Health Organ. 2005; 85:100-8.

7. Monteiro CA, Mondini L, Costa RBL. Mudanças na composição e adequação nutricional da dieta familiar nas áreas metropolitanas do Brasil (1988-1996). Rev Saúde Pública. 2000; 34:251-8.

8. Schoeller D. How accurate is self-reported dietary energy intake? Nut Rev. 1990; 48:373-9.

9. Endo SK, Carmo HCE. Pesquisa de Orçamentos Familiares no município de São Paulo. São Paulo: Instituto de Pesquisas Econômicas, Universidade de São Paulo; 1984.

10. Kirsten JT, Berndt A, Moraes CNP, Alves ELG, Carletti JA, Rubião MNS, et al. Orçamentos familiares na cidade de São Paulo 1971/1972. São Paulo: Instituto de Pesquisas Econômicas; 1973.

11. Carmo HCE. Pesquisa de Orçamentos Familiares 98-99: principais resultados. Informações FIPE. 1999; 231(12):12-7.

12. Instituto Brasileiro de Geografia e Estatística. Pesquisa de Orçamentos Familiares 2002/2003: análise da disponibilidade domiciliar de alimentos e do estado nutricional no Brasil. Rio de Janeiro; 2004.

13. Instituto Brasileiro de Geografia e Estatística. Estudo Nacional das Despesas Familiares-ENDEF: 1974-1975. Rio de Janeiro; 1978.

14. Becker W. Comparability of household and individual food consumption data - evidence from Sweden. Public Health Nutr. 2001; 4:1177-82.

15. Drewnowski A. Fat and sugar: an economic analysis. J Nutr. 2003; 133:838s-40s.

16. Drewnowski A, Specter SE. Poverty and obesity: the role of energy density and energy cost. Am J Clin Nutr. 2004; 79:6-16.

Recebido em: 24/10/2006

Versão final reapresentada em 3/5/2007 Aprovado em: 8/8/2007 